\title{
Reviewer Acknowledgements for Global Journal of Health Science, Vol. 7, No. 1
}

Global Journal of Health Science wishes to acknowledge the following individuals for their assistance with peer review of manuscripts for this issue. Their help and contributions in maintaining the quality of the journal is greatly appreciated.

Global Journal of Health Science is recruiting reviewers for the journal. If you are interested in becoming a reviewer, we welcome you to join us. Please find the application form and details at http://www.ccsenet.org/reviewer and e-mail the completed application form to gjhs@ccsenet.org.

\section{Reviewers for Volume 7, Number 1}

Alexander Domnich

Ama Pokuaa Fenny

Amir Sayem

Ammar Eltayeb Ali Hassan

Anjan Ghosh

Asad Ali Khan Afridi

Azadeh Saki

Bashiru Saeed

Brian Cook

Caroline Elizabeth Bulsara

Daniel Weber

Darampal Dambhare

Dhairya Lakhani

Domitila Augusta Huber

Emad Shdaifat

Evangelia Mavrikaki

Francisco Rodenas Rigla

Gabriele Messina

Hadii Mamudu

Jacob D. Christenson

Jason Tsai

Kartheek R Balapala

Kimberley Geissler

Kingly Wangdi

Le Thi Thanh Xuan

Loray Daws

Majid Naderi
Mark Tully

Mcgrowder

Mehdi Jafari

Meng Zhao

Montarat Thavorncharoensap

Myo Nyein Aung

Nazisa Hejazi

Patricia Ruell

Pavlos Sarafis

Polly Yeung

Qilong Wang

Raywat Deonandan

Rod Macleod

Samendra Sherchan

Samira Schultz Mansur

Sara Melo

Sherif Hassan

Shilpee Singh

Somayeh Noori Hekmat

Srikrishna Sulgodu Ramachandra

Tarek Tawfik Amin

Trisha Dunning

Wenhua Lu

William Cho

Yevgeniy Samyshkin

Zada Pajalic

Zahra Fazli-Khalaf 